\title{
Development of CFRP racing motorcycle rims using a heuristic evolutionary algorithm approach
}

\section{Journal Article}

\section{Author(s):}

Giger, M.; Ermanni, P.

Publication date:

2005

\section{Permanent link:}

https://doi.org/10.3929/ethz-b-000158283

\section{Rights / license:}

In Copyright - Non-Commercial Use Permitted

\section{Originally published in:}

Structural and Multidisciplinary Optimization 30(1), https://doi.org/10.1007/s00158-004-0471-3 


\title{
Development of CFRP racing motorcycle rims using a heuristic evolutionary algorithm approach
}

\author{
M. Giger and P. Ermanni
}

\begin{abstract}
The scope of this paper is the application of evolutionary optimization methods to the development of composite fibre reinforced plastics (CFRP) racing motorcycle rims. The mass and the moment of inertia of a front and a rear CFRP rim are minimized subject to manufacturing, strength, and stiffness constraints. The stacking sequence of the composite laminates is optimized by applying a sophisticated parameterization concept making an excellent compromise between a huge variety of structure properties and a reasonable number of optimization parameters. The mechanical properties are simulated using the finite element analysis package ANSYS. Resulting displacement and Tsai-Wu index values are combined with the mass of the rim in order to assign a fitness value to each different design solution. The smart formulation of the fitness function allows the exploration of solutions close to the required strength and stiffness properties. The proprietary software DynOPS is utilized as an optimization engine. It links an evolutionary algorithm to arbitrary simulation programs and controls the entire optimization process. The sophisticated parameterization concept, together with the fitness function formulation, are the basis for the development of CFRP motorcycle rims decisively lighter than state-of-the-art magnesium alloy rims.
\end{abstract}

Key words evolutionary design optimization, structural optimization, composite laminate optimization, parameterization, evolutionary algorithms

Received: 22 June 2004

Revised manuscript received: 26 July 2004

Published online: 26 January 2005

(C) Springer-Verlag 2005

M. Giger ${ }^{-}$and P. Ermanni

Centre of Structure Technologies, Institute of Mechanical Systems, Swiss Federal Institute of Technology, Zurich, Switzerland

e-mail: giger@imes .mavt.ethz.ch,

ermanni@imes.mavt.ethz.ch
1

\section{Introduction}

In motorcycle racing it is absolutely crucial to permanently pursue improvements and new developments in order to remain competitive. A variety of components contribute to an outstanding racing performance, e.g., engine performance, aerodynamic properties, wheel grip, the drivers' skills, but in particular the motorcycle rims significantly influence the overall performance.

The quality of a motorcycle rim can be directly quantified through its mechanical properties such as stiffness and strength. The stiffness is particularly important in turns of high speed where vertical and lateral loads resulting from the motorcycle's weight and centripetal effects introduce bending loads into the rear and the front rim. Furthermore, the motorcycle rims must not fail when maximum loading occurs. The front rim is heavily loaded when full braking is applied. The maximum loading of the rear rim occurs at maximum acceleration of the motorcycle once the front rim loses ground contact.

In addition to the mechanical properties, the mass and the moments of inertia of a motorcycle rim should be as low as possible. On the one hand, the rim mass contributes to the overall mass of the motorcycle and it belongs to the so-called unsprung mass crucially influencing the handling of the motorcycle on uneven race tracks. On the other hand, low moments of inertia of the rims are much more important. As a matter of simple physical principles, a lower moment of inertia with respect to the rotation axis of the rim allows faster acceleration and deceleration of the wheels and therefore of the racing motorcycle. Moreover, changing the direction of the motorcycle creates gyroscopic forces whose magnitudes are directly correlated to the moments of inertia of the wheels. It is therefore crucial to reduce the moments of inertia - in particular of the front rim - in order to improve the handling, the performance, and therefore the competitiveness of the racing motorcycle.

Nowadays, magnesium alloy rims that are even lighter than aluminium rims are state-of-the-art in motorcycle racing. These magnesium alloy rims are technologically 
sophisticated and therefore do not offer a great potential of further mass reduction. Thus, the newly developed rims are manufactured from carbon fibre reinforced plastics (CFRP) allowing for a significant reduction of mass and moments of inertia due to superior stiffness and strength properties of the material.

The rims are manufactured by combining unidirectional and woven CFRP plies, whereas the mechanical properties and the orientation of each ply directly influence the mechanical properties of the rim structures. Consequently, the highly complex issue of determining the optimum stacking sequence of the plies needs to be addressed. Only the application of advanced optimization methods allows for the consequent exploration of the mass reduction limits. A characteristic for composite optimizations are numerous local minima in the search space, which need to be anticipated. Furthermore, the integer numbers describing the quantity of plies and the discrete parameters determining the orientation of the plies render the search space extremely discontinuous. Additionally, a variety of constraints have to be considered during the optimization in order to produce feasible design solutions. Consequently, the optimization problem cannot be efficiently solved by using gradient-based optimization techniques so that evolutionary optimization methods need to be chosen.

A basic introduction to the optimization methodology based on evolutionary algorithms (EA) is presented in Sect. 2. Section 3 gives a brief overview of the required steps to apply EAs to the rim optimization problem. The setup of the necessary simulation models is outlined in Sect. 4, whereas the entire optimization process is the subject of Sect. 5. Within the scope of this project, a front and a rear rim are developed. The methodology of the optimization process is demonstrated by means of the front rim but the optimization results are presented for both motorcycle rims in Sect. 6. Finally, a short conclusion and an outlook are given in Sect. 7.

\section{2}

\section{Basic concepts of evolutionary algorithms}

The idea behind evolutionary algorithms $(\mathrm{EA})^{1}$ is to imitate Darwin's "survival of the fittest" law within an artificial simulation environment. Similarly to nature, where every species evolves through the mating of the fittest individuals, different physical structures performing well under a given load case are combined in order to find an even better structure. This is not done by directly combining the physical structures themselves, but by combining their determining parameter values, similar to the combination of two parents' genes that form the genes of a child. This way the problem is divided into a phenotype solution space (the physical structures) and a genotype search space (the coded solutions), which is named

\footnotetext{
${ }^{1}$ For a definition of terms see Bentley (1999)
}

the representation issue by Schoenauer (1996) or alternatively the parameterization in this paper.

In structural optimization the applied evolutionary algorithms are most often based on genetic algorithms (GAs). GAs were developed by Holland (1975) and the basic terminology of genetic search and its principal components are discussed by Goldberg (1989). An introduction to the application of GAs to structural optimization using traditional binary string codings is presented by Hajela (1992). A more modern overview of the application of evolutionary algorithms in automated design optimization can be found in Bentley (1999).

\section{Basic elements of general EAs}

This section briefly recalls the basic steps of a general EA, mostly following the terminology introduced by Bentley (1999). The problem at hand is to reach an objective within a given search space subject to some limiting constraints. A fitness function has to be built from these quality measures (optimization objective and constraints) assigning a unique fitness value to each individual, whereas violated constraints should significantly affect this fitness value. A population of individuals undergoes artificial Darwinian evolution whereby the fitness value is directly related to the parameter-set of the respective individual. The best individuals of a population are used to produce offspring for the subsequent population, i.e., the next generation. Figure 1 shows a rough scheme of an evolutionary algorithm paradigm and the main steps are described below.

Initialization. An initial population of individuals is required in order to start the optimization process. Such a population can be obtained by randomly initializing the genotype, whereas each entry of the genotype, i.e., each single gene, can be chosen from a given set of possible parameter values (alleles).

Evaluation. Every individual of a population has to be rated according to the fitness function formulation. This includes transformation of the individual from

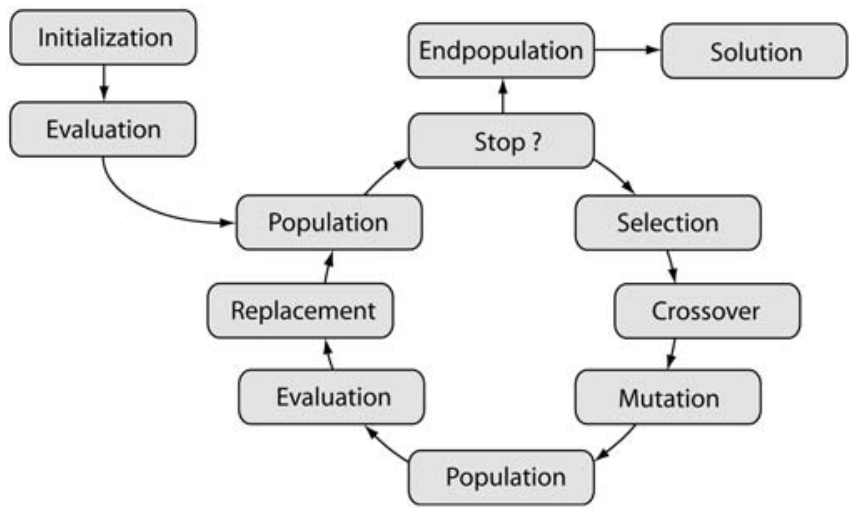

Fig. 1 Basic elements and optimization loop of an evolutionary algorithm 
the search space (genotype) to the solution space (phenotype) and the calculation of the quality measure, i.e., the fitness value, which is most often realized by finite element analysis (FEA). In structural optimization the computation of the fitness values causes most of the CPU costs.

Selection. In each iteration of the optimization loop a set of individuals is chosen from the latest population and put into the genitor pool to generate offspring individuals. This process is called selection, whereas different selection methods based on stochastic or deterministic principles can be used. As an example, the deterministic tournament selection compares the fitness values of some randomly chosen individuals from the population and then selects the $n$ best individuals among them to put them into the genitor pool. This procedure is repeated until the required number of individuals in the genitor pool is reached. This kind of selection process gives strong individuals a high probability to be genitor for the next generation but also weaker individuals are not completely neglected. According to Hammel and Bäck (1998) this applies a medium selection pressure to the population and the variety does not decrease too much.

Crossover. From the genitor pool, offspring individuals are generated by applying so-called crossover operators. The genetic information of two genitor individuals are recombined to generate new offspring individuals. A variety of different crossover operators exist, whereas for example the uniform, intermediate, onepoint, two-point, segment, and hypercube crossovers can be applied.

Mutation. Mutation increases the variety in the newly created offspring by modifying single genes of some randomly chosen individuals. Uniform mutation selects parameter values from all possible alleles according to a uniform distribution, whereas the Gaussian mutation is based on a selection scheme following a Gaussian probability distribution. Mutation is important to escape from local minima of the fitness landscape in case all individuals are situated around such local minima.

Replacement. A new population is built by deterministic or stochastic choice of parents and offspring.

The newly created population is then evaluated again and the optimization loop starts anew.

Stopping criterion. The entire process of evaluation, reproduction, and replacement continues until a given stopping criterion is reached. This can, for example, be based on a time or generation counter or the best fitness of the population is compared to a target value that has to be reached. The stopping criterion can, alternatively, be based on the convergence behaviour of the optimization process itself, i.e., the optimization is halted when a given number of generations does not produce an improved solution. Then it is assumed that the optimization process is converged to an at least local optimum solution.

\section{3}

\section{Evolutionary optimization methods applied to the rim optimization problem}

Figure 2 illustrates a general working schedule for structural optimization problems addressed with an evolutionary approach.

Before evolutionary algorithms can be used for the optimization of any structural part, some preparatory steps have to be carried out. First, a simulation model of the structure to be optimized is required in order to evaluate the fitness value for each individual. A CAD-model of the motorcycle rim is prepared using the CAD-software CATIA V5 ${ }^{2}$ defining the geometric shape of the structure (Sect. 4.1). In this project the geometric shape of the motorcycle rim remains unchanged during the optimization process, only the stacking sequence of the composite laminate is altered. The CAD-model forms the basis of a finite element (FE) model generated in $\mathrm{ANSYS}^{3}$ used to evaluate stiffness, strength, and mass properties of the motorcycle rim (Sect. 4.2).

After defining the simulation model, the optimization process can be set up. The so-called parameterization (Sect. 5.1) is the key to an efficient and successful optimization. It has to be defined which parameters, e.g., the number of plies at a given position or the orientation of a certain ply, are modified during the optimization process. Obviously, there are thousands of possibilities to parameterize a laminated structure. The structure can be subdivided into an arbitrary number of sections having different stacking sequences and therefore requiring a lot of optimization parameters defining the number of fibre plies and their orientation. The number of optimization parameters defines the size of the genotype and accordingly the size of the search space. A large number of parameters expands the search space and generally requires a greater number of evaluations to explore the search space sufficiently and to finally converge to an optimum solution. Therefore, it is extremely important to choose an appropriate parameterization that can produce reasonable optimization results. The last step before starting the optimization process is the definition of the

\footnotetext{
2 www.catia.ibm.com

3 www.ansys.com
}

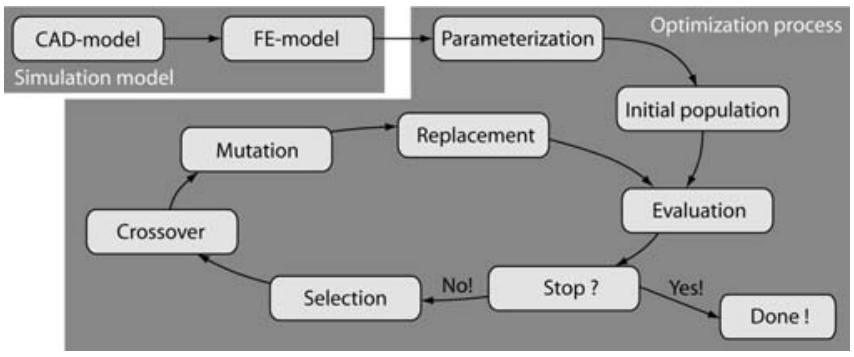

Fig. 2 Working schedule of evolutionary algorithms applied to structural optimization problems 
evaluation step. A fitness function (Sect. 5.2) mapping the evaluated stiffness, strength, and mass measures from the FE-simulation to a unique fitness value needs to be defined.

After these preparatory steps, the actual optimization process can be started (Sect. 5.3). A starting population is initialized based on random initialization.

Afterwards, the optimization loop containing evaluation, selection, crossover, mutation, and replacement is iteratively run until a given stopping criterion is reached and an optimum design solution is found.

In the following sections all the above mentioned steps are discussed in detail.

\section{4}

\section{Simulation model}

For both the front and the rear rim a FE-simulation model has to be set up. Since this project is concerned with the complete new development of the motorcycle rims, CAD-models only defining the geometric shapes, but no material or other structural properties, are created first. Then FE-models defining the stacking sequences are derived from these CAD-models. Finally, the load cases for the simulation of the mechanical behaviour are defined.

\section{1}

\section{CAD-model}

The CAD models define the geometric shapes of the motorcycle rims and are therefore heavily constrained to meet a variety of requirements. There are some interfaces to adjacent parts that have to comply with small tolerances in order to guarantee faultless function. Probably the most important interface is situated between the tires and the rims since it is there that all acceleration and deceleration forces are transmitted through frictional forces. The geometric shapes of the rim beds are therefore given by the geometry of the tires. Furthermore, the front rim has two more interfaces to the brake disks transmitting the braking forces over the tires to the race track. The rear rim has only one interface to a brake disk, but additionally, the drive torque is transmitted from the engine via the chain through five rubber inserts to the rear rim. Finally, the rims have bearing carriers forming the connection to the motorcycle suspensions.

Only the spokes and adjacent regions of the hub are left to the designer's creativity, but still constraining factors must be considered. The width of the spokes is limited by the design space boundaries given through the brake caliper.

It is decided to use a five-spokes design, particularly due to the customer acceptance, but also stiffness properties, load transmission qualities, etc., are not less important. The bending stresses introduced by maximum drive or brake torque are reduced by connecting the spokes tan-
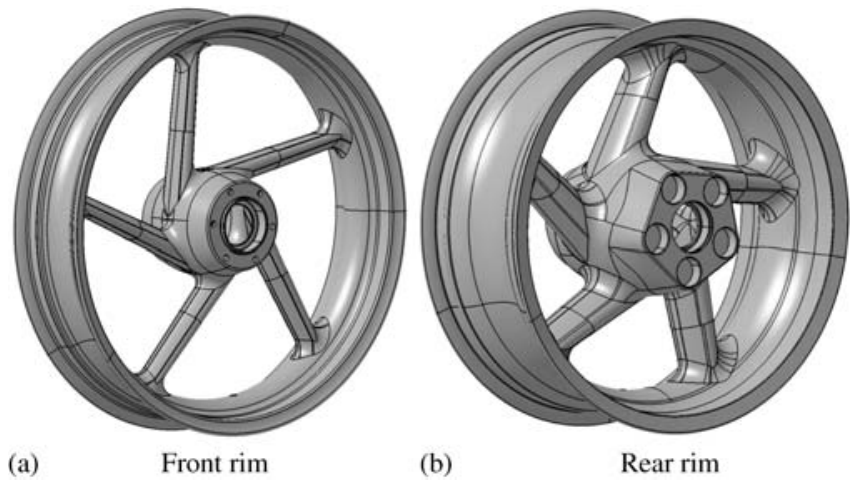

Fig. 3 CAD models

gentially to the hub. The design of the rims, and in particular the spokes, are defined without running shape optimizations, since at this stage the optimization software tools have not allowed for a combined optimization of geometric shape and stacking sequence of the laminates.

\section{2 \\ FE-model}

The FE-models of the front and rear rims are set up in ANSYS by importing surface models from the CADsoftware CATIA V5. The surface model is then meshed with layered 4-node isoparametric shell elements (SHELL181) admitting the definition of laminate properties, i.e., the stacking sequence consisting of single CFRP plies.

The purpose of the FE-model is to simulate the critical load cases occurring during a race and to prove the mechanical strength of the rims. Furthermore, the FEanalysis is also applied in order to estimate the stiffness properties of the rims. The rims must not be too compliant even if they would not fail due to mechanical stresses. Consequently, two load cases per front and rear rim have to be defined, one simulating the maximum load case and another providing a stiffness measure. For brevity, only the FE-model of the front rim is discussed. Both load cases for the front rim are illustrated in Fig. 4.
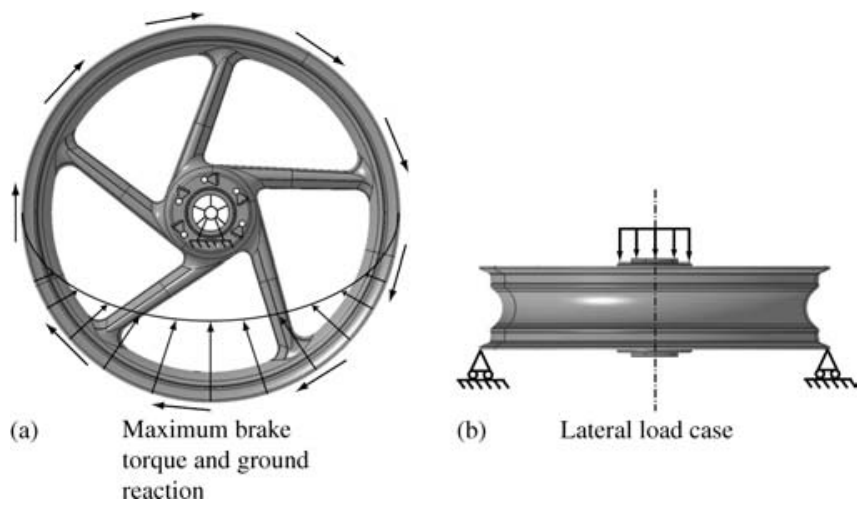

Fig. 4 FE model load cases of the front rim 
Maximum loading of the front rim occurs in full braking stages when the rear wheel loses ground contact. All the braking forces are transmitted through the front rim to the race track causing a torsional loading of the structure.

The brake torque is assumed to be equally distributed along the circumference of the part of the rim bed where it is in direct contact with the tire. At the bearing carrier the rim is axially and laterally supported and the holes for the bolted joints to the brake disk carriers are supported to block the rotational degree of freedom. Additionally, the ground reaction force has to be superposed such that it is multiplied with a scaling factor in order to take vertical impacts into account. The ground reaction force is assumed to follow a cosine distribution along the lower half of the rim circumference. In former projects (Giger 2001) this assumption produced the most accurate simulation results compared to practical deformation tests. For this load case, lateral forces introduced by centripetal effects can be neglected.

For the estimation of the stiffness properties a lateral load case is evaluated. The rim is laterally supported at one outer rim bed and a lateral force is applied on the hub. The deflection value is compared to results from tests with state-of-the-art magnesium alloy rims. The final aim is to approximately match the stiffness properties of these.

\section{5}

\section{Optimization process}

Based on the FE model of the front rim the optimization process is set up by defining the parameterization of the rim model in Sect. 5.1 and introducing a fitness formulation for the rating of the evaluated rim individuals in Sect. 5.2. Within Sect. 5.3 the software tool DynOPS controlling the optimization loop is briefly explained.

\section{1}

\section{Parameterization}

The parameterization links the search space (genotype) to the solution space (phenotype). The model parameters spanning the search space and simultaneously determining the appearance of the model in the solution space are defined. Since the geometric shape of the motorcycle rim is fixed, no shape parameters appear. Only parameters defining the stacking sequence of the composite laminate are admissible.

\subsection{1}

\section{Optimization parameters}

The choice of optimization parameters determines how the stacking sequence of the rim can be changed during the optimization process in order to find an optimum design solution. Basically, there are three different pa- rameters describing the stacking sequence of a structural part made from CFRP prepregs, i.e., carbon fibres impregnated with thermosetting epoxy resins.

- A variety of prepreg materials are available with different fibre layouts. In this project a unidirectional carbon laminate and woven carbon laminates are used, whereas the woven fabric is available in two different thicknesses.

- The number of fibre plies has to be determined.

- The orientation of each orthotropic ply needs to be defined.

The representation of these properties is addressed by using a heterogeneous list of optimization parameters.

\subsection{2}

\section{eoUniGene - the heterogeneous genotype}

The evolving objects ${ }^{4}$ (EO) framework freely available under the GNU Lesser General Public licence provides the basic tools to perform any kind of evolutionary strategies within the field of evolutionary computing. On the basis of this open-source project, a universal genotype named eoUniGene, able to handle heterogeneous lists of parameters in an efficient way by applying appropriate operators, was developed by König (2004) and Wintermantel (2003). This universal genotype consists of a collection of different gene types that can be combined to a heterogeneous genotype.

A variety of different gene types are available within eoUniGene, whereas only four gene types are used for the parameterization of the motorcycle rims.

- String-list gene is a list of arbitrary discrete values upon which no norm or ordering can be applied.

- Boolean gene represents a Boolean parameter that can be true or false.

- Const-float-list gene represents equidistant floating-point values. Upper and lower limit value can be provided and the number of intervals in between is to be defined.

- Integer gene represents an integer parameter, whereas lower and upper limit as well as the stepsize need to be provided.

These gene types are used for the parameterization of the stacking sequences of the composite laminates. For each ply, the Boolean, the string-list, and the const-float-list gene need to be defined.

- A maximum number of plies is defined for the stacking sequence in each section of the rim. The Boolean gene determines whether a ply of the stacking sequence is active (true) or not (false). By setting the Boolean value to false for some plies they can be omitted to reduce the rim mass. This procedure is necessary because the evolutionary operators always require equal length genotypes. It is therefore impossible to only

\footnotetext{
4 http://eodev.sourceforge.net, Keijzer et al. (2001)
} 


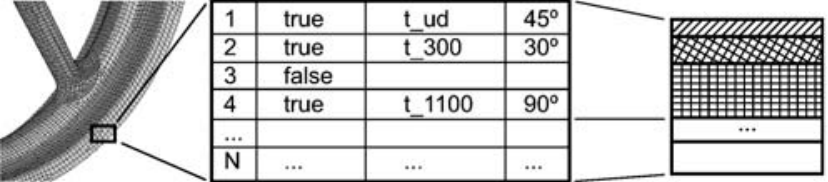

Fig. 5 Schematic illustration of the parameterization concept

represent all existing plies and to remove the genes defining the plies that are eliminated from the genotype.

- The string-list gene identifies the material of a ply. For the manufacturing of the motorcycle rim three different prepreg types are selected. The first prepreg type is a standard unidirectional carbon laminate with a thickness of $0.15 \mathrm{~mm}$ represented by the string $t_{-} u d$. The second and third prepreg types are woven carbon laminates (orientation $0^{\circ}$ and $90^{\circ}$ ) having thicknesses of $0.25 \mathrm{~mm}$ and $0.5 \mathrm{~mm}$, respectively. They are represented by the strings $t_{-} 300$ and $t_{-} 1100$ according to their product names. ${ }^{5}$ All these materials are defined in the FE-model and can therefore arbitrarily be chosen by the optimization engine.

- The third gene type, the so-called const-float-list gene, determines the orientation of the chosen prepreg for each ply. Due to the limited manufacturing accuracy of approximately $5^{\circ}$ it is useless to admit arbitrary orientation angles, since then the search space would be enormously large. Thus, only a few possible values (alleles) for the ply orientation are taken into account. These values need to span the whole range from $-90^{\circ}$ to $90^{\circ}$ in order to tap the full potential of the CFRP materials. The range is subdivided into twelve equidistant sections leading to possible ply orientation values of $-90^{\circ},-75^{\circ},-60^{\circ}$, to $60^{\circ}, 75^{\circ}$, and $90^{\circ}$. In fact, the range for the woven laminates only needs to span a range from $0^{\circ}$ to $90^{\circ}$, but the parameterization does not distinguish between two different ranges for unidirectional and woven laminates since the laminate type is chosen independently from the ply orientation. The increment of $15^{\circ}$ is larger than the theoretically possible manufacturing accuracy, but it is considered to be sufficiently small to not lose a potentially best solution.

The integer gene is only needed in a few sections to locally define the number of additional unidirectional reinforcement plies with a $0^{\circ}$ orientation. Figure 5 illustrates schematically the parameterization of an arbitrary section.

\section{1 .3}

\section{Optimization model}

Obviously, the stacking sequence should not be identical for the entire rim model since there are many differently

${ }^{5}$ LTM26-EL component prepregs. http://www.acg.co.uk stressed parts of the structure. It is important to only utilize as much fibre material as absolutely necessary in each section of the rim in order to tap the full potential of mass and moment of inertia reduction. Consequently, the rim structure has to be subdivided into several sections having different stacking sequences. The boundaries of these sections are defined in consideration of the force flow in the structure, enabling the optimization to locally reinforce highly stressed regions by applying additional plies. The number of sections directly influences the number of optimization parameters because each section with a different stacking sequence requires additional optimization parameters. In consideration of the rule of thumb that the number of parameters to be optimized should not exceed the population size to get reasonable optimization performance beyond pure stochastic search, the number of different sections cannot be arbitrarily high. In consideration of the available computing power, a maximum of approximately sixty to eighty optimization parameters is desired.

It is decided to subdivide the rim structure into four major domains showing different stacking sequences of the composite laminates. The first domain includes the hub and the spokes, the second domain incorporates the rim bed, and the third domain defines the region where the first two domains overlap each other. Domain number four has the same base laminate as the first domain, but some additional unidirectional plies are added in order to locally reinforce the highly stressed region, i.e., the transition zones between the hub and the spokes. Figure 6 illustrates the subdivision into these four domains with the respective optimization parameters.

For the first two domains, base-composite laminates are defined consisting of a maximum number of ten plies

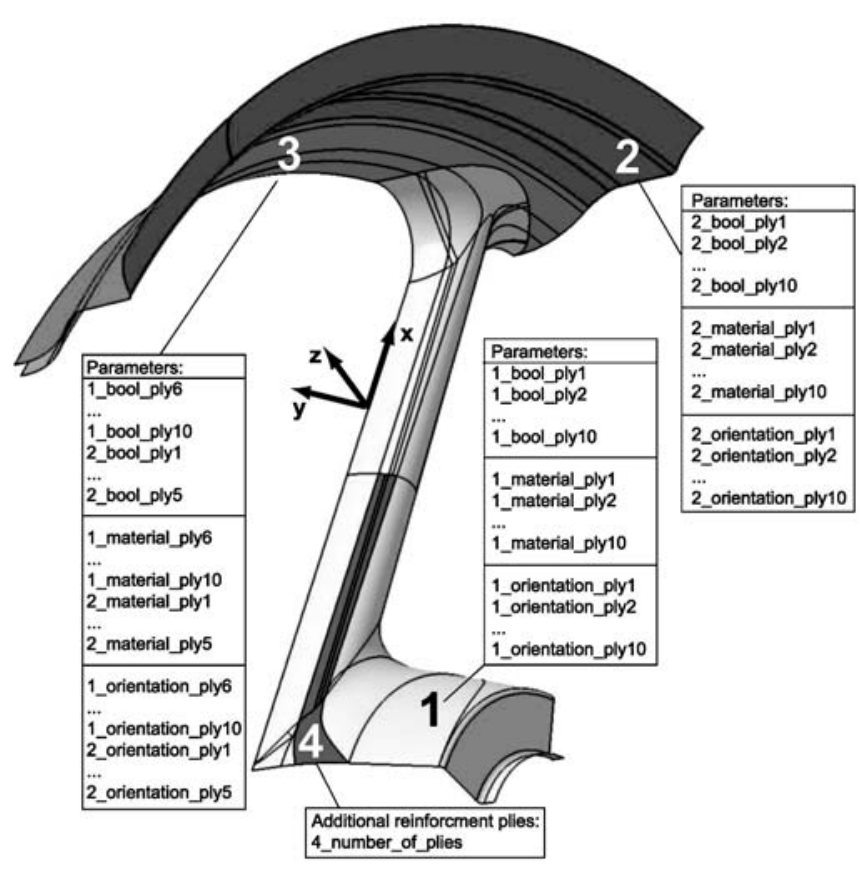

Fig. 6 Schematic illustration of the four major domains 
each. The choice of the maximum number of ten plies is based on an antecedent finite element simulation using woven laminates only leading to a feasible design solution. Both base-composite laminates require 30 parameters in order to define their stacking sequences. Ten parameters determine whether each of the ten plies is active or not, ten parameters define the prepreg material, and ten further parameters define the ply orientations. In the third domain, both base laminates are partially overlapped in order to guarantee a proper connection between the first two domains, hence, no additional parameters are required. For the fourth domain, an integer parameter is added to the genotype to define the additional number of unidirectional reinforcement plies.

The optimization model finally consists of 61 parameters to be optimized in order to achieve the optimization objective, i.e., minimization of the structural mass in consideration of stiffness and strength constraints.

For the purpose of rating the individuals, a fitness function needs to be defined combining the optimization objective with the optimization constraints.

\section{2}

\section{Fitness function}

The optimization objective is to minimize the front rim's mass and accordingly its moment of inertia. Simultaneously, two constraints have to be fulfilled, i.e., the rim must not collapse under maximum loading and it has to provide a target stiffness for sufficient handling properties. The strength criterion is based on the well known Tsai-Wu criterion (Tsai and Wu 1971) for laminated structures. The Tsai-Wu index must remain below the limit value of 1 in order to prevent damage to the structure. The stiffness criterion relies on practical stiffness tests with magnesium alloy rims, whereas the CFRP rim should approximately achieve the same stiffness properties for the lateral load case as outlined in Sect. 4.2. The lower deflection limit is set to $0.13 \mathrm{~mm}$ and the upper limit is set to $0.23 \mathrm{~mm}$. The optimization can be formulated as follows:

\section{Minimize Mass}

$$
\begin{aligned}
& \text { subject to: } \quad \text { Tsai-Wu index }<1 \\
& 0.13 \mathrm{~mm}<\text { Deflection value }<0.23 \mathrm{~mm}
\end{aligned}
$$

The objective and both constraints are mapped to a single fitness value $F(\mathbf{p})$ to rate the quality of every evaluated individual represented by the parameter vector $\mathbf{p}$ by building a weighted sum of the form

$F(\mathbf{p})=\sum_{i} w_{i} D_{i}(\mathbf{p})$

where $D_{i}(\mathbf{p})$ represents the rating for the objective or a specific constraint and $w_{i}$ is the corresponding relative weight. This means that every objective and constraint value computed during the fitness evaluation must be mapped to an addend $D_{i}$ of the fitness function. The mapping is done in order to assign a fitness portion to each constraint in the interval $[0,1]$. Only the optimization objective is allowed to exceed this range for low quality individuals. The contribution of all fitness portions to the overall fitness value can be controlled by defining the relative weights $w_{i}$. For the optimization of the front rim the relative weights are left to 1 . It is essential that high quality design solutions lead to lower fitness values than comparatively worse solutions. Thus, this optimization is a minimization problem where the absolute minimum of the fitness $F(\mathbf{p})$ should be found.

\section{Mapping functions $D_{i}$}

For the optimization, objective and constraints mapping functions are utilized that were introduced by König (2004) and proved to be of value in a variety of former optimization problems. Only the mapping functions of the front rim are presented, the mapping functions of the rear rim are analogously defined with slightly differing parameter values. All the parameter values defining the mapping functions are based on experience.

Optimization objective. The mapping function for the optimization objective, i.e., the reduction of the rim's mass, is generally formulated as

$D_{i}(O)=(a O+b)^{\alpha}$,

where $O$ is the mass of the rim to be minimized. The choice of the exponential factor $\alpha=5$ is based on experience, and $a$ and $b$ are scaling factors defined by the conditions

$D_{i}\left(O=O_{\text {init }}\right)=1$

$D_{i}\left(O=O_{\text {estim }}\right)=0.1$,

where $O_{\text {init }}$ represents an initial value of the design objective that should result in a fitness value of 1 . In the case of a front rim optimization this value is set to 1500 grams. $O_{\text {estim }}$ is the estimated value that is expected to be reached through the optimization process corresponding to a fitness value of 0.1 . In this case this value is set to 900 grams. Figure 7 illustrates the mapping function for the optimization objective leading to fitness values in the interval $[0, \infty[$. Since the FE-model is simplified compared to the CAD-model, the evaluated mass of each individual is below the effective mass of the corresponding manufactured rim. This difference does not affect the optimization itself, because the fitness portion of the mass is a relative measure for the quality of the rim.

Strength constraint. The mapping function of the strength constraint is implemented using a smooth step function to keep solutions with Tsai-Wu index values close to the limit of 1 in the population. The definition of this mapping function is as follows

$D_{i}(C)=\frac{1}{1+e^{-\lambda\left(C(\mathbf{p})-C_{\text {limit }}-\Delta\right)}}$, 


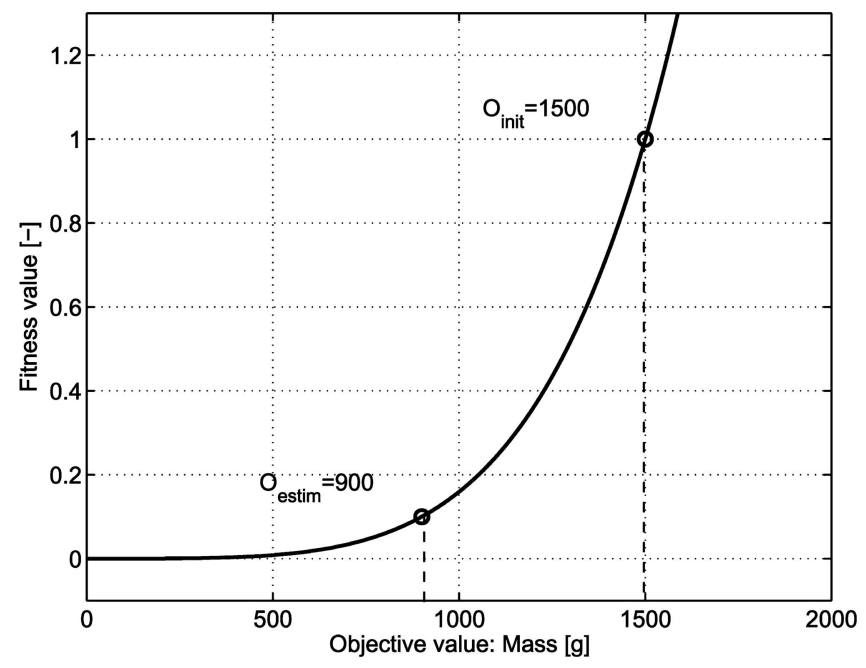

Fig. 7 Mapping function for the optimization objective (mass)

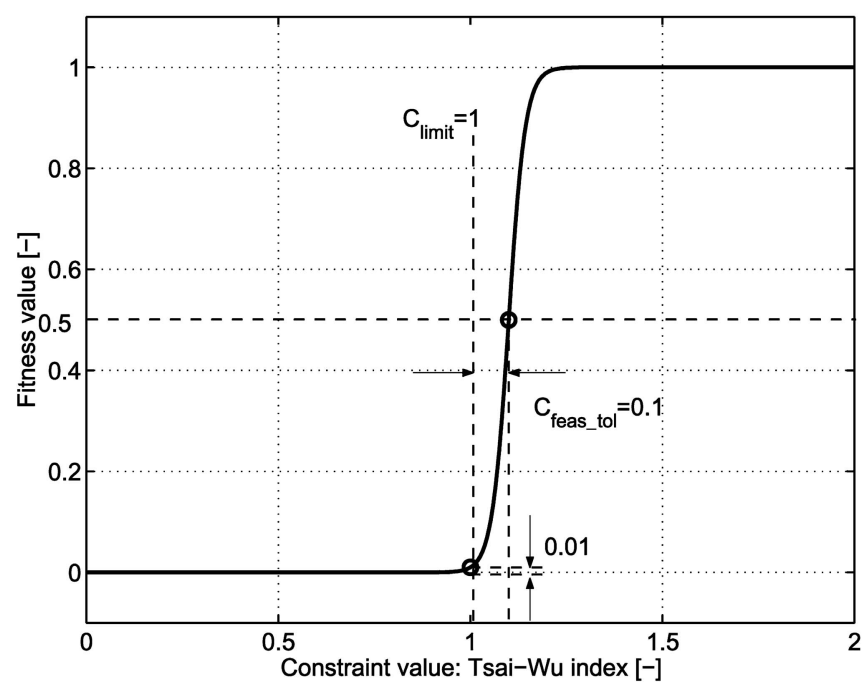

Fig. 8 Mapping function for the upper limit constraint (Tsai-Wu index)

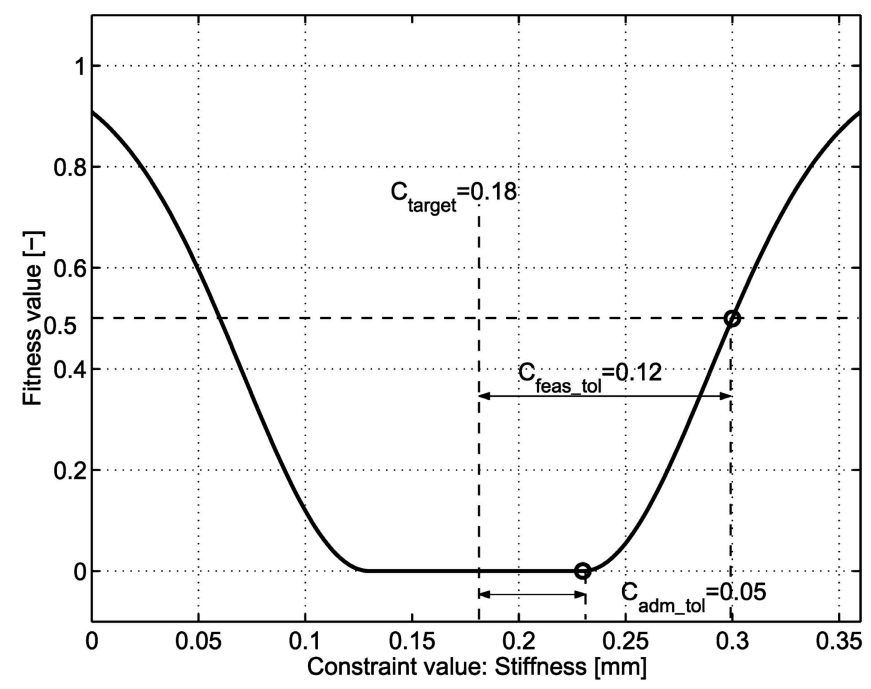

Fig. 9 Mapping function for the target constraint (stiffness) where the following functions define the parameters $\lambda$ and $\Delta$ :

$$
\begin{aligned}
& \lambda=\frac{1}{C_{\text {feas_tol }}}\left(\ln \left(\frac{1}{D_{\text {limit }}}-1\right)-\ln \left(\frac{1}{D_{\text {feas }}}-1\right)\right) \\
& \Delta=\frac{1}{\lambda} \ln \left(\frac{1}{D_{\text {limit }}}-1\right) .
\end{aligned}
$$

The shape of the mapping function can be adjusted by adapting the values $D_{\text {limit }}$ and $D_{\text {feas }}$ :

$D_{i}\left(C_{\text {limit }}\right)=D_{\text {limit }}=0.01$

$D_{i}\left(C_{\text {limit }}+C_{\text {feas_tol }}\right)=D_{\text {feas }}=0.5$.

Finally, only the parameter values $C_{\text {limit }}$ and $C_{\text {feas_tol }}$ need to be altered in order to tune the strength constraint. Figure 8 illustrates the mapping function used for the optimization of the front rim.

Stiffness constraint. The mapping function of the stiffness constraint should direct the optimization process to the given target value of $0.18 \mathrm{~mm}$, and at least to be within the aforementioned limits. Consequently, the lower and the upper limit have to be considered in the function definition. The shape of the mapping function is therefore a symmetric step function, whereas the values of the function around the target value are zero. The definition of the mapping function requires three parameter values, i.e., $C_{\text {target }}, C_{a d m_{-} \text {tol }}$, and $C_{\text {feas_tol }}$. Figure 9 illustrates the meaning of these values. The formulas defining the mapping function are as follows:

$D_{i}(C)= \begin{cases}0 & : c_{d}<C_{a d m_{-} t o l} \\ 1-e^{-\frac{\left(\left|C(\mathbf{p})-C_{\text {target }}\right|-C_{a d m_{-} t o l}\right)^{2}}{2 \sigma^{2}}}: & c_{d} \geq C_{a d m_{-} t o l}\end{cases}$

where the absolute difference from the target value is given by

$c_{d}=\left|C(\mathbf{p})-C_{\text {target }}\right|$.

The mapping function definition is finally completed by the following two values depending on the previously introduced parameter values

$\sigma^{2}=\frac{\left(C_{\text {feas_tol }}-C_{\text {adm_tol }}\right)^{2}}{-2 \ln \left(1-D_{\text {feas }}\right)}$

and

$D_{i}\left(C_{\text {target }}+C_{\text {feas_tol }}\right)=D_{\text {feas }}=0.5$.

The width and the steepness of this mapping function are critical parameters, because poorly initialized optimization runs may completely miss the target value.

\section{3 \\ DynOPS controlled optimization loop}

The execution of the optimization process is based on a variety of components. The EO library, the parameter- 
ization based on eoUniGene, the evaluation of the FE model for each individual, and the assignment of a fitness value to the evaluated individuals needs to be managed. A proprietary software tool called DynOPS (Dynamic Optimization Parameter Substitution) written in $\mathrm{C}++$ is able to cope with this complex task. DynOPS is a very general tool able to connect the EO library with any simulation software that can be started in batch-mode, e.g., ANSYS, CATIA V5, etc. The evaluation of the individuals during the optimization loop is most often computationally expensive. For this reason DynOPS runs the evaluation tasks in parallel by distributing them to a potentially heterogeneous hardware environment. DynOPS consists of four different modules.

- Optimization engine. The EO library provides the necessary framework to apply evolutionary algo- rithms in general. In connection with the heterogeneous genotype eoUniGene, see Sect. 5.1.1, an optimization engine for structural optimization problems is established.

- Text file interface. DynOPS creates text-based input files to run arbitrary simulation software (in this project ANSYS for the evaluation of all individuals) in their batch mode. A prototype text file has to be provided with the specification of the optimization parameter positions within this file. DynOPS then generates new input files based on the new parameter values provided by the optimization engine that are sent to the simulation software for the evaluation of the respective individual. After these individuals are evaluated, DynOPS reads the result values, i.e., objective and constraint values, from output text files created by the simulation software in order to evalu-

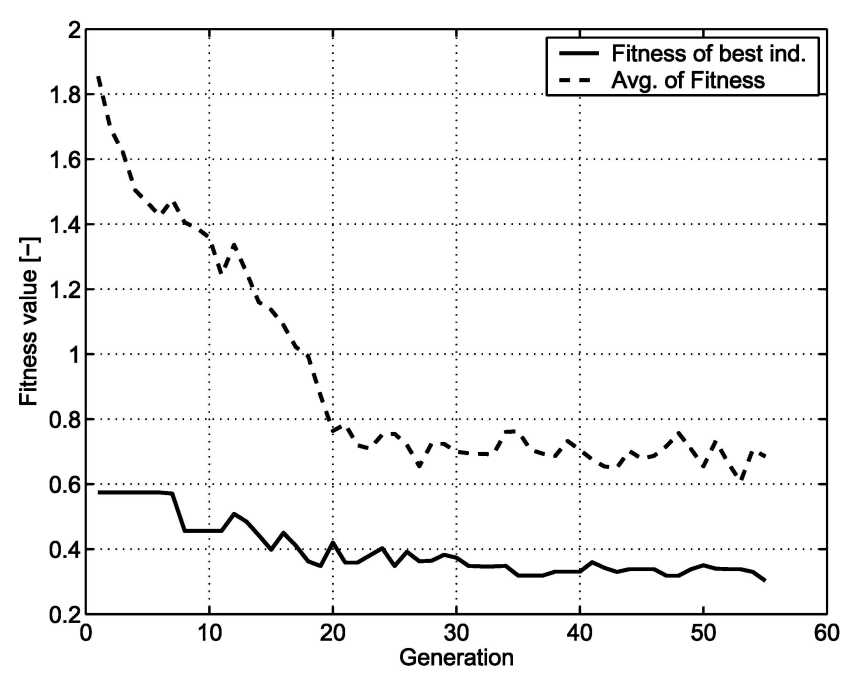

(a)

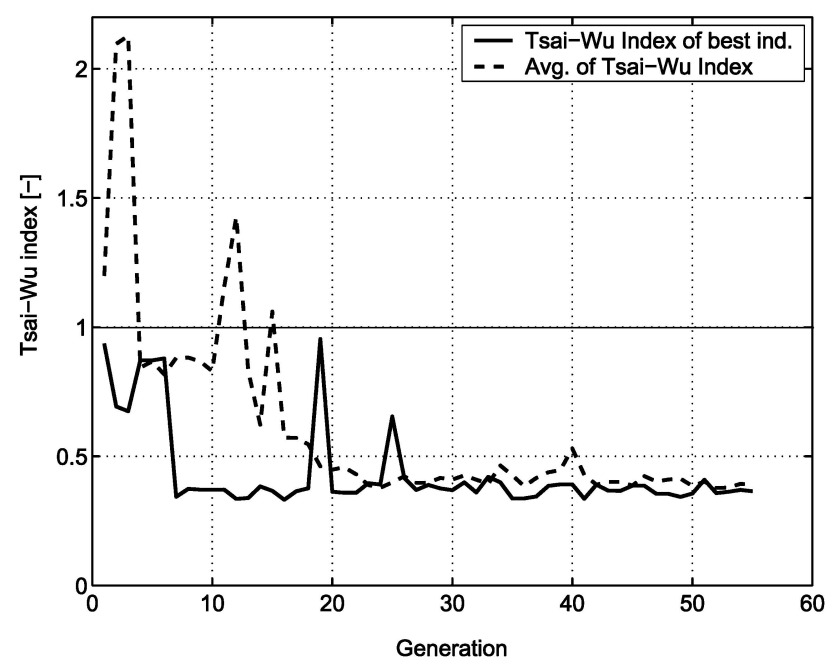

(c)

Tsai-Wu index

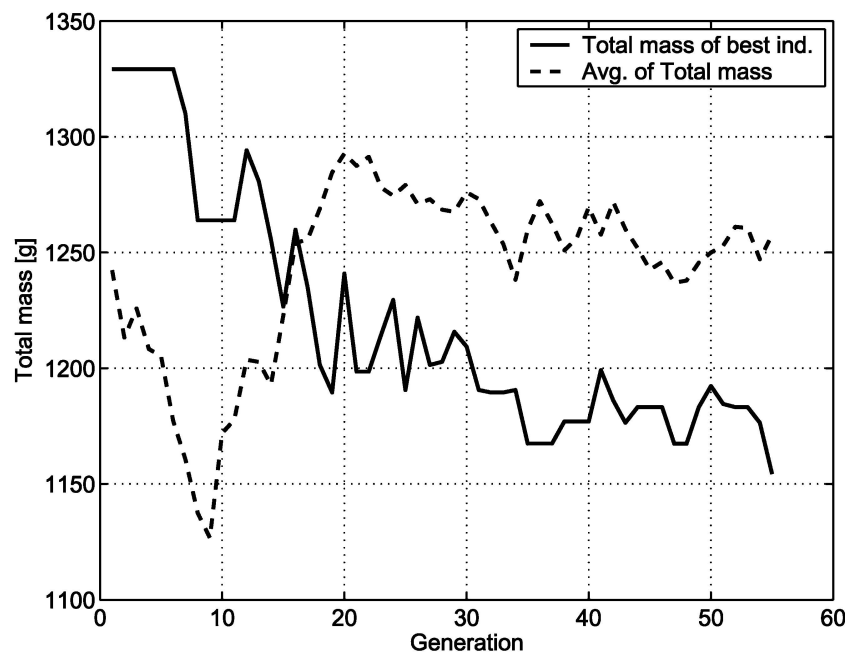

(b)

Mass

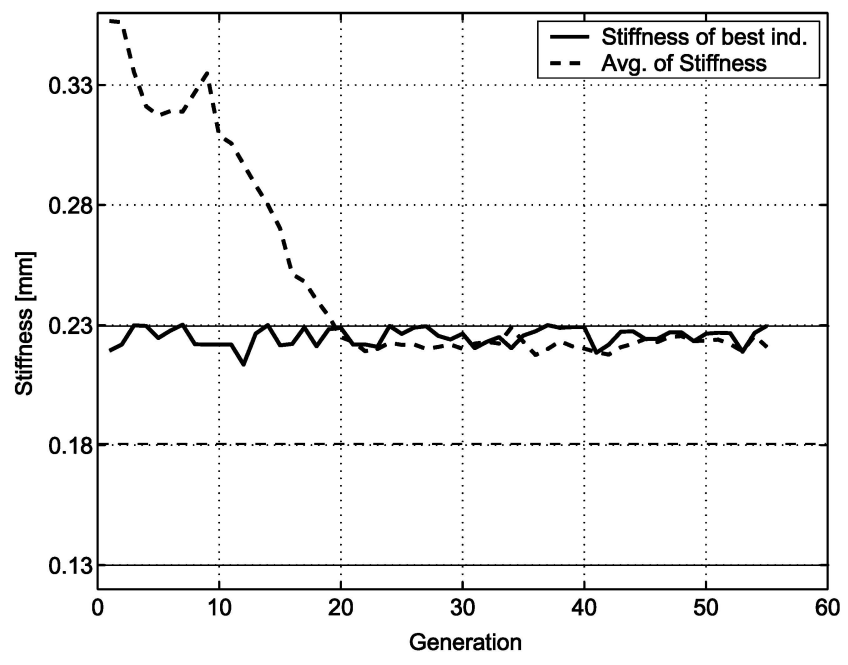

(d)

Fig. 10 Convergence plots of the front rim optimization 
ate the overall fitness value according to the defined fitness function.

- Process manager. The process manager specifies a sequence of programs with their adequate input files to be executed during the optimization process, whereas the input and also the output files can be in any data format. It is possible to use files of arbitrary format, e.g., a modified CAD geometry that is passed to a finite element analysis.

- Parallelization. DynOPS is able to evaluate the individuals of a population in parallel using the parallel virtual machine (PVM) library. ${ }^{6}$ The parallel machine is set up based on the user-specified list of processors that need to be connected, whereas DynOPS

${ }^{6}$ http://www.csm.ornl.gov/pvm/pvm_home.html

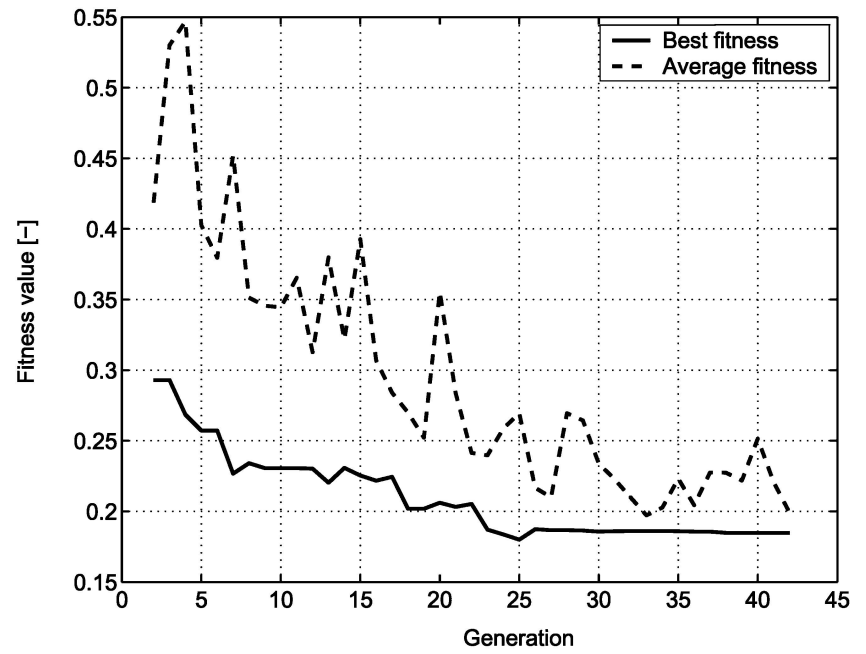

(a)

Fitness value $F(\mathbf{p})$

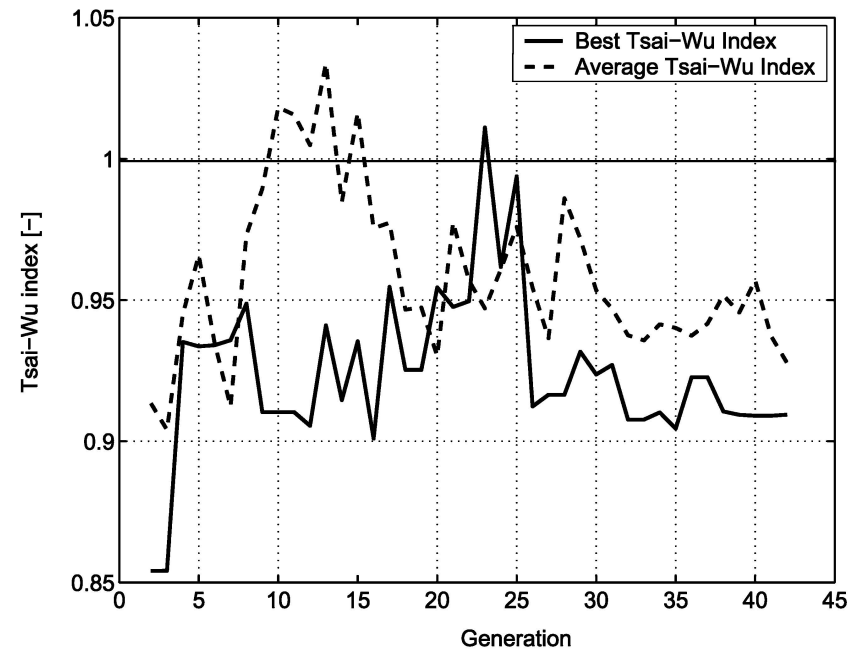

(c)

Tsai-Wu index

Fig. 11 Convergence plots of the rear rim optimization manages the evaluation tasks in a queue and distributes them to available processors.

For more detailed information on DynOPS see Wintermantel (2003).

\section{6 \\ Optimization results}

Figure 10 illustrates some convergence plots of the front rim optimization. In each plot the objective and constraint values of the best individual having the lowest overall fitness value $F(\mathbf{p})$ within the population of a generation as well as the average value of the entire population are presented. The plots show the first 55 evaluated generations; further evaluations have not found significantly improved design solutions and are therefore omit-

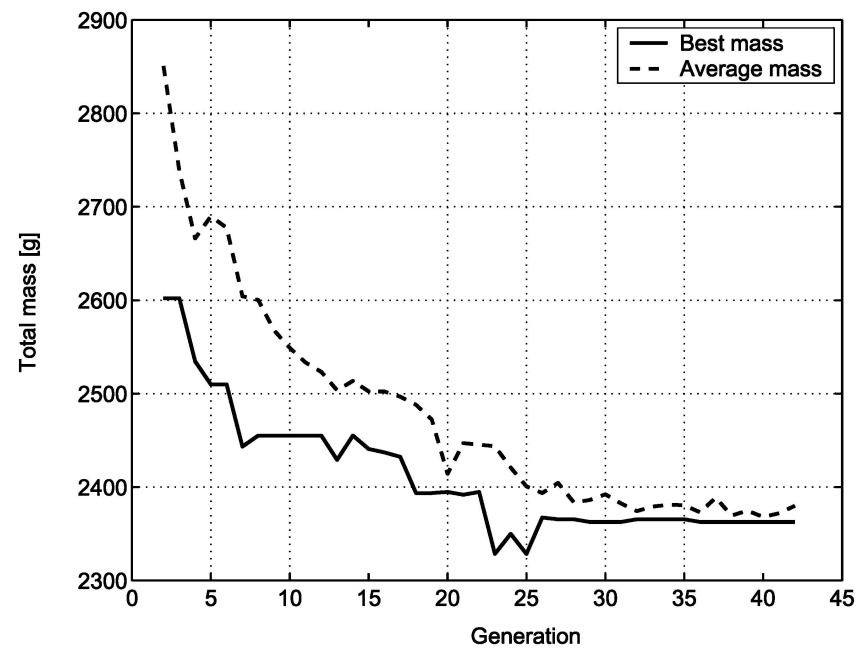

(b)

Mass

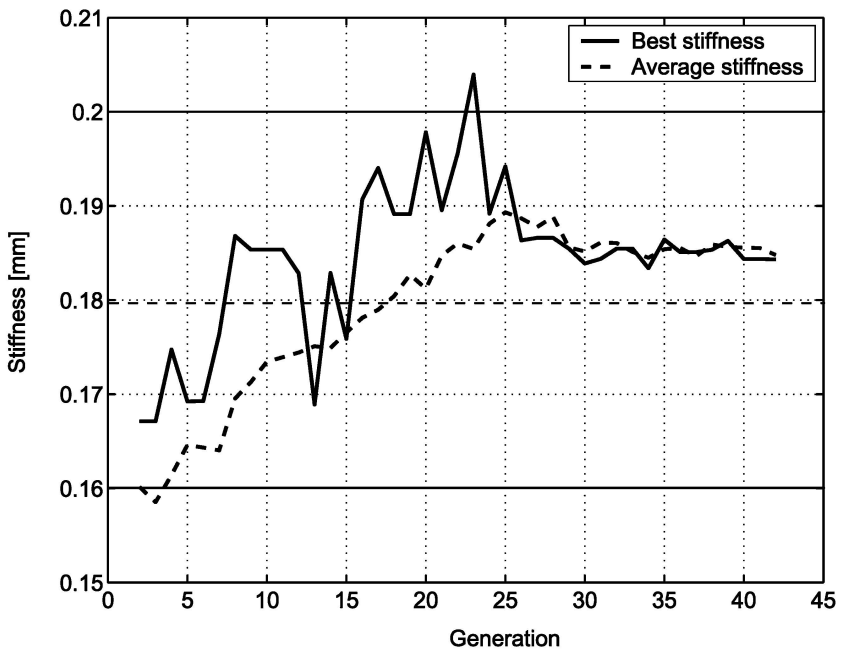

(d)

Stiffness value 
ted from the plots. The optimization run is performed with 50 individuals per generation since the computing time is also a kind of an optimization constraint. This number is slightly below the rough rule of thumb saying that the number of individuals per generation should be at least the number of optimization parameters (61). Instead, the probability of mutation is chosen rather high in order to ensure a sufficient exploration of the entire search space.

Regarding the convergence plots it is important to note that from the beginning of the optimization process, the respective best solutions always fulfil both the strength and the stiffness constraint. The overall fitness value is reduced to approximately $50 \%$ of the initial fitness value, whereas the mass of the best individual is reduced by about $15 \%$. The average of the fitness value is reduced simultaneously indicating a decreasing diversity in the populations. Obviously, these results are heavily influenced by the chosen parameterization. If the number of independent domains would be chosen much higher, e.g., ten instead of 4 , there would be an even greater potential of mass reduction. The stacking sequences could be adjusted more precisely to the loading in each domian, but then the optimization would be computationally more expensive since this implicates a larger search space. Furthermore, it is extremely important to establish a parameterization leading to feasible designs from the beginning of the optimization, otherwise the optimization might be incapable of finding any feasible design solution. Finally, only the stiffness constraint restricts the optimization since the optimum rim is manufactured from a lot of thick woven layers to achieve the stiffness target value. The optimization of the front rim clearly shows that it is the stiffness constraint that prevents the rim from becoming lighter. Under the given load cases the front rim is not at risk to fail due to high stresses (TsaiWu index of 0.4).

The results of the rear rim optimization are presented in Fig. 11. Basically, the results are similar to the optimization results of the front rim optimization. The geometric shape of the rear rim is different from the front rim shape and therefore another parameterization and slightly different mapping functions are defined. The optimization is also run with 50 individuals per generation
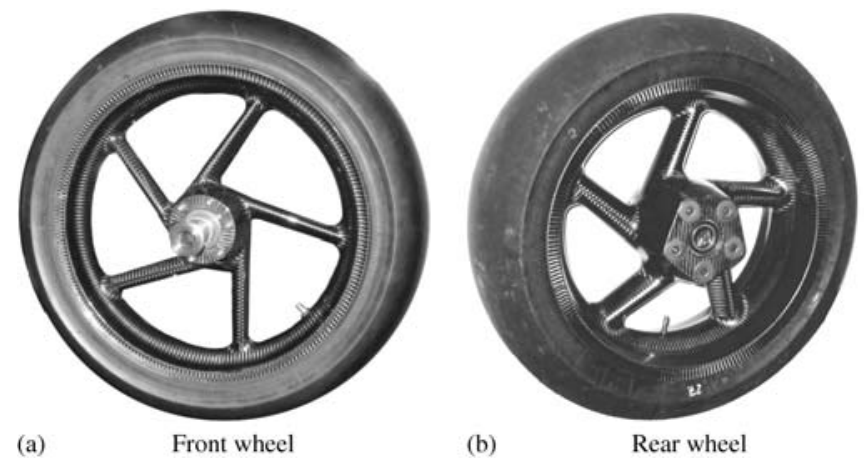

Fig. 12 Manufactured rim prototypes and is stopped after 42 generations. The fitness value of the best individual in each generation is reduced by approximately $38 \%$, whereas the mass is reduced by about $9 \%$. The rear rim optimization finds an optimum design solution where the strength and the stiffness constraint are quite close to their allowable limits.

Both optimizations finally lead to manufacturable stacking sequences of the laminates consisting of all three given materials. The ply orientation angles span the range from $-90^{\circ}$ to $90^{\circ}$ whereas some directions are particularly reinforced according to the force flow in the respective sections. The maximum number of plies is not utilized in all sections of the rim, i.e., the mass of the rims is reduced by choosing small prepreg materials and by eliminating entire plies from the stacking sequence.

The optimization results are the basis for the realization of both motorcycle rims. Details of the manufacturing process are not discussed within the scope of this paper.

\section{7 \\ Conclusion and outlook}

Evolutionary algorithms are a well-suited tool for the optimization of laminated structures. The presented paper demonstrates their applicability particularly for problems with discrete search spaces by using the sample problem of motorcycle rims heavily constrained by shape and manufacturing constraints. Only the application of the heterogeneous genotype eoUniGene and an adequate parameterization technique make this optimization possible and successful. The formulation of the fitness function consisting of smooth constraint mapping functions steers the optimization into an appropriate direction, finally leading to significantly improved CFRP motorcycle rims that could hardly be designed by human intuition.

A major drawback of the optimization at hand is that the evolutionary optimization requires equal-length genotypes. This disadvantage has to be overcome by introducing a Boolean gene for each ply that decides whether the ply is omitted or not. Even if a certain ply is removed the defining genes for the choice of material and orientation remain in the genotype and are transmitted to the next generation, although they do not influence the phenotype solution. Thus, the optimization efficiency and the solution quality might be affected adversely. The further research will therefore be focussed on the development of variable-length genotypes and their appropriate operators in order to improve the quality of optimization.

Acknowledgements This work was supported by the KTI/CTI Innovation Promotion Agency. Basic research results applied in this project emerged from the project "DesignEntity based Structural Optimization with Evolutionary Algorithms" (SNF 21-66879.01) supported by the Swiss National Science Foundation. 


\section{References}

Bentley, P.J. 1999: Evolutionary design by computers. San Francisco, CA: Kaufmann

Giger, M. 2001: Strukturanalyse einer Formel-1-Felge. Term project IMES-ST-Nr 02-036, Centre of Structure Technologies, Institute of Mechanical Systems, ETH Zürich

Goldberg, D.E. 1989: Genetic algorithms in search, optimization and machine learning. Reading, MA: Addison-Wesley

Hajela, P. 1992: Stochastic search in structural optimization: genetic algorithms and simulated annealing. In: Kamat M.P. (ed.) Structural optimization: status and promise. Progress in astronautics and aeronautics series, vol. 150, pp. 611-637

Hammel, U.; Bäck, Th. 1998: Optimierung in der Simulation: Evolutionäre Algorithmen. Technical Report, Sonderforschungsbereich 531 Computational Intelligence, University of Dortmund

Holland, J.H. 1975: Adaptation in natural and artificial systems. Ann Arbor, MI: University of Michigan Press
Keijzer, M.; Merelo, J.J.; Romero, G.; Schoenauer, M. 2001: Evolving objects: a general purpose evolutionary computation library. In: EA-01, Evolution Artificielle, 5th International Conference in Evolutionary Algorithms, pp. 231-244

König, O. 2004: Evolutionary design optimization: tools and applications. Dissertation, Swiss Federal Institute of Technology, Zurich, Diss. ETH No. 15486

Schoenauer, M. 1996: Shape representations and evolution schemes. In: Fogel, L.J.; Angeline, P.J.; Back, T. (eds.), Fifth Annual Conference on Evolutionary Programming. Boston: MIT Press, pp. 121-129

Tsai, S.W.; Wu, E.M. 1971: A general theory of strength for anisotropic materials. J. Compos. Mater. January, 58-80

Wintermantel, M. 2003: Design-encoding for evolutionary algorithms in the field of structural optimization. Dissertation, Swiss Federal Institute of Technology, Zurich, Diss. ETH No. 15323 